\title{
Mental health risk factors influencing metabolic syndrome among secondary school teachers of Mysore city, India
}

\author{
Shashikala Narayanappa $^{1}$, Renuka Manjunath ${ }^{2}$, Praveen Kulkarni ${ }^{2}$
}

Department of Community Medicine, Mysore Medical College Research Institute, Mysore, India

Department of Community Medicine, JSS Medical College, Mysore, India

Received: 28 March 2016

Revised: 08 April 2016

Accepted: 11 April 2016

\section{*Correspondence:}

Dr. Shashikala Narayanappa,

E-mail: shashiaklarm@yahoo.com

Copyright: $\odot$ the author(s), publisher and licensee Medip Academy. This is an open-access article distributed under the terms of the Creative Commons Attribution Non-Commercial License, which permits unrestricted non-commercial use, distribution, and reproduction in any medium, provided the original work is properly cited.

\section{ABSTRACT}

Background: Metabolic syndrome has been suggested to be an intermediate pathway between depression and CVD wherein, chronic stress due to prolonged exposure to work stress, repeated stress over a period of time damages the HPA axis resulting in a maladaptive process as well as inflammatory factors like CRP which causes depression and poor health habits, leading to development of metabolic syndrome. The risk factors causing stress among the school teachers is very high as compared to other professional groups which not only affects their health but also adversely affects the students and their learning environment. The interventions have been mainly to reduce the level of work related stress and improve their performance in teaching, but have neglected a holistic approach to improve their overall health conditions.

Methods: Cross sectional study was done in Mysore city among 320 Secondary school teachers aged 20 years and above, with at least one year of teaching experience. A self-administered, pretested and structured questionnaire based on the WHO steps approach for NCD, Gurmeet Singh's Presumptive Stressful Life Events Scale (PSLES) for stress, PHQ 9 for depression and GHQ28 for general mental health status.

Results: The prevalence of metabolic syndrome was 115 (38.3\%). 30.6\% had mild to moderate depression and only 2.3\% had severe depression. $141(47.0 \%)$ had moderate stress and only $42(14 \%)$ had severe stress. The prevalence of minor psychiatric disorders (MPD) was $66(22 \%)$. The mental health status did not have any statistically significant effect on the occurrence of MS or its risk factors.

Conclusions: The poor mental health status was significantly associated with high fasting blood glucose levels, moderate to severe depression was significantly high among females and stress was associated with diastolic blood pressure, TGs and dyslipidemia.

Keywords: Metabolic syndrome, Stress, Depression, Risk factors, Screening

\section{INTRODUCTION}

The metabolic syndrome is a cluster of abnormalities such as abdominal obesity, hypertension, dyslipidemia, (increased triglycerides and reduced HDL-C) and glucose intolerance which have been associated with increased risk for subclinical atherosclerosis, CVD (two fold, RR of 1.5-2.9), T2DM, target organ damage and total mortality. ${ }^{1-3}$ All these are components of metabolic syndrome act as intermediate risk factors in the pathway for CVD and T2DM. They have $30-40 \%$ probability of developing DM and/or CHD in 20 years. ${ }^{3,4}$ The risk of incident and death due to CHD is about 1.5 to 2.88 in people with metabolic syndrome when compared to nonmetabolic syndrome. The multiple risk factors increase the risk more than the sum of each component. ${ }^{5}$ 
Metabolic syndrome has also been suggested to be an intermediate pathway between depression and CVD wherein, chronic stress due to prolonged exposure to work stress, repeated stress over a period of time damages the HPA axis resulting in a maladaptive process as well as inflammatory factors like CRP causes depression and poor health habits leading to development of metabolic syndrome. ${ }^{6,7}$

Depression is a complex condition characterized by disruptions in all facets of life-social, psychological, behavioural and biological. The psychological stress is associated in a dose response manner with metabolic syndrome however, extreme stressful life events related to finance and chronic work stress, are more likely to have the metabolic syndrome than other stressful events. ${ }^{8-}$ 10 Stress also induce some psychosocial factors like change in sleep pattern and depression which dysregulate the HPA axis and ANS system affecting the neuro endocrine and autonomic function by increasing the cortisol (activating the HPA axis), catecholamine's and IL-6 output respectively and predisposing to metabolic syndrome. ${ }^{7,9,10}$ Thus, chronic psychological stress, depression and obesity result in the pathophysiological changes which reduces resilience and disturbs homoeostasis resulting in insulin resistance obesity, increased TGs, IGT and systolic/diastolic blood pressure and finally metabolic syndrome..$^{9,10}$

Studies across the world have revealed that the prevalence of stress and psychiatric morbidity to be very high among school teachers as compared to other professional groups which, has adverse effects on health resulting in reduces quality of life as well as adversely affects the students and their learning environment. ${ }^{11-13}$ Also, the prevalence of metabolic syndrome varies from $11 \%$ to $41 \%$ in India, depending on the definition used ethnicity of the population and place of residence. ${ }^{14}$

Considering the level of work stress among teachers and the association of stress and depression with metabolic syndrome, the present study was conducted with the objectives of studying the influence of stress and depression on metabolic syndrome and its components among secondary school teachers.

\section{METHODS}

This cross sectional study was done in Mysore city, with 213 secondary schools and 1924 secondary school teachers working in these schools. 300 secondary school teachers aged 20 years and above, with at least one year of teaching experience and who gave consent to participate in the study were included.

Sample size was estimated based on CURES 2006 Chennai study which reported a prevalence of $25.8 \%$ Metabolic syndrome according to International Diabetic federation (IDF) criteria. $^{8}$ Relative allowable error of $20 \%$ was considered and the sample was estimated to be
288 , adding $10 \%$ of non-response the final sample was 320. Off which 300 subjects were considered for analysis as their information was complete.

Multistage sampling technique was adopted where, in first step all the secondary schools in Mysore city were stratified into, Government, private aided and private unaided schools and from each of these strata teachers were selected by probability proportionate to size technique.

A self-administered, pretested and structured questionnaire based on the WHO Steps Approach for NCD evaluation was used to assess the sociodemographic profile, disease profile, level of physical activity and habits (smoking, alcohol, diet). The blood pressure and anthropometric measurements including weight, height and waist measurements were obtained using standard techniques. ${ }^{15}$ The fasting blood sample was taken for analyzing fasting blood sugar, triglycerides and HDL cholesterol levels.

Patient Health Questionnaire 9 (PHQ- 9) was used to determine level of depression. The nine items of the PHQ-9 are based directly on the nine diagnostic criteria for major depressive disorder in the DSM-IV (diagnostic and statistical manual fourth edition). Each domain has 4 subscales (0-3). No depression (0-4), mild depression (59), moderate depression (10-19) and severe depression $(>20){ }^{16,17}$

Stressful life events in the past year were determined using the Gurmeet Singh's presumptive stressful life events scale (PSLES), which is an Indian adaptation of the original Holmes and Rahe's social readjustment rating schedule. The scale has adequate psychometric properties and local norms. It gives quantitative estimate of presumptive stress (weighted scores) experienced by Indian adult population. The scores assigned to each individual item varying from $0-100$ and then ranked according to severity. The total scores were obtained by adding all the applicable life event scores. The scores were then categorized into no stress (0-40), mild to moderate stress (41-200) stress and severe stress $(>200){ }^{18}$

GHQ 28 was used to determine the mental health status (developed by Goldberg and Hiller 1979). It measures the common mental health domains of depression, anxiety, somatic symptoms and social withdrawal. Each domain is composed of four subscales (0-7). The reliability coefficient (Cornbach's alpha) is 0.95 for GHQ 28. ${ }^{19,20}$ the Scoring criteria of GHQ 28 is normal $(<23)$ and poor mental health status $(>23)$.

The institutional ethics committee clearance, permission of the regional Deputy Director of Public Instructions DDPI, principals and the written informed consent of the teachers was obtained before starting the study. 
Metabolic Syndrome was defined based on the IDF consensus 2005 criteria. Central obesity measured as waist circumference $(>80 \mathrm{~cm}$ in females and $>90 \mathrm{~cm}$ in males of Asian origins), is central to the diagnosis along with any two of the following criteria if present was characterised as metabolic syndrome:

1) Fasting blood glucose $\geq 100 \mathrm{mg} / \mathrm{dl}$ or previously diagnosed as diabetic,

2) Hypertension $\geq 130 / 85 \mathrm{mmHg}$ or previously diagnosed as hypertensive,

3) Dyslipidemia

i) Triglycerides $\geq 150 \mathrm{mg} / \mathrm{dl}$ and/ or,

ii) HDL C $\leq 40 \mathrm{mg} / \mathrm{dl}$ in males and $\leq 50 \mathrm{mg} / \mathrm{dl}$ in females or previously diagnosed as dyslipidemia or on medication for the same. ${ }^{2}$

\section{Statistical analysis}

Data collected was entered in MS Excel 2010 and analyzed using SPSS version 20. Descriptive statistical measures such as mean, standard deviation for continuous variables and number and percentage for categorical variables were used. Comparison between continuous variables was done using independent " $t$ " test and for categorical variables by using chi square test. Binary logistic regression analysis was done to see the factors which were independent predictors of metabolic syndrome. The differences and associations were interpreted as statistically significant at $\mathrm{P}<0.05$.

\section{RESULTS}

\section{General characteristics}

Among the study population $112(37.3 \%)$ and 188 $(62.7 \%)$ were males and females respectively. The average age of the teachers was $44.7 \pm 9.5$ years, 253 $(84.3 \%)$ were married and had $15.2 \pm 9.0$ years of teaching experience. $58(19.3 \%), 97(32.3 \%)$ and $145(48.3 \%)$ the teachers belonged to government, private aided and private unaided schools respectively. Most of the teachers $195(65 \%)$ of them lived in nuclear families.

\section{Metabolic syndrome and the risk factors}

The prevalence of metabolic syndrome was $38.3 \%$ (115) according to the IDF definition 2005. The prevalence of metabolic syndrome among males was $38.4 \%$ and females was $41.5 \%$, the difference was statistically not significant $(p>0.05)$. The prevalence of metabolic syndrome increased with age from $14.3 \%$ in age group $21-30$ years to $56.3 \%$ in $>50$ years age group $(\mathrm{p}<0.001)$.

Obesity (68.6\%) and low HDL-C (39.4\%) were significantly higher in females $(\mathrm{p}<0.01)$. However, hypertension $(59.8 \%)$, hypertriglyceridemia $(179.54 \pm 88.4$ $\mathrm{mg} / \mathrm{dl})$ and physical activity (16.1\% and $29.4 \%$ intense and moderate activity respectively) was higher in males $(\mathrm{p}<0.001)$.

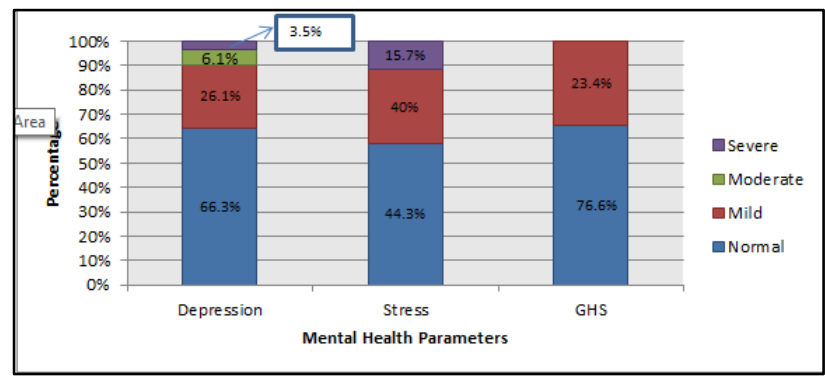

Figure 1: Distribution of subjects according to mental health status $(n=300)$.

\section{Mental health status and metabolic syndrome}

The prevalence of mild psychiatric disorders (MPD) was in $66(22 \%)$, mild to severe depression in $99(33 \%)$, moderate stress in $141(47 \%)$ and severe stress in 42 (14\%) among the subjects. The present study did not show any statistically significant association between the presence of mental health parameters and metabolic syndrome. However, the prevalence of mild psychiatric disorders (MPD) and any form of depression was significantly higher among females $(\mathrm{p}<0.05)$, whereas level of stress was similar in both males and females.

Table 1: Socio demographic characteristics of the study population.

\begin{tabular}{|lllll|}
\hline Variable & Total, $\mathbf{N}=\mathbf{3 0 0}$ & Male $(\mathbf{1 1 2})$ mean, SD & Females $(\mathbf{1 8 8})$ mean, SD & Test of significance \\
\hline Age & 44.7 years \pm 9.5 & 44.7 years \pm 9.53 & 41.2 years \pm 10.2 & 0.788 \\
\hline Marital status & $253(84.3 \%)$ & $100(89.3 \%)$ & $153(81.4 \%)$ & 0.077 \\
\hline Years of teaching & 15.2 years \pm 9.0 & 17.6 years, 8.8 years & 13.76 years, 8.8 years & 0.465 \\
\hline $\begin{array}{l}\text { Type of school } \\
\text { Government }\end{array}$ & $58(19.3)$ & $18(16.1)$ & $40(21.3)$ & 0.000 \\
Private aided & $97(32.3)$ & $53(47.3)$ & $44(23.4)$ & \\
Private unaided & $145(48.3)$ & $41(36.6)$ & $104(55.3)$ & 0.379 \\
\hline $\begin{array}{l}\text { Type of family } \\
\text { Nuclear }\end{array}$ & $195(65)$ & $76(67.8)$ & $119(63.3)$ & \\
Others & $105(35)$ & $36(32.1)$ & $69(36.7)$ & \\
\hline
\end{tabular}


Table 2: Biophysical profile, and mental health status of the study population.

\begin{tabular}{|c|c|c|c|c|}
\hline Variable & Total, $n=300$ & Male(112) mean, SD & Females(188) mean, SD & Test of significance \\
\hline $\begin{array}{l}\text { Physical activity } \\
\text { Intense activity } \\
\text { Moderate activity } \\
\text { Sedentary }\end{array}$ & $\begin{array}{l}20(6.7) \\
77(25.7) \\
203(67.7)\end{array}$ & $\begin{array}{l}18(16.1) \\
33(29.4) \\
61(54.5)\end{array}$ & $\begin{array}{l}2(1.1) \\
44(57.4) \\
142(75.5)\end{array}$ & 0.001 \\
\hline $\begin{array}{l}\text { Obesity (WC) } \\
\text { Normal } \\
\text { Obese }\end{array}$ & $\begin{array}{l}110(36.7) \\
190(63.3)\end{array}$ & $\begin{array}{l}51(45.5) \\
61(54.5)\end{array}$ & $\begin{array}{l}59(31.4) \\
129(68.6)\end{array}$ & 0.014 \\
\hline BMI & $25.7 \pm 4.9$ & $24.32 \pm 3.16$ & $26.55 \pm 0.41$ & 0.000 \\
\hline Systolic blood pressure & $123 \pm 15.9$ & $128.45 \pm 13.43$ & $120.58 \pm 16.41$ & 0.01 \\
\hline $\begin{array}{l}\text { Diastolic blood pressure } \\
\text { Triglycerides }\end{array}$ & $\begin{array}{l}80.1 \pm 10.2 \\
149 \pm 78.4\end{array}$ & $\begin{array}{l}83.9 \pm 9.24 \\
179.54 \pm 88.4\end{array}$ & $\begin{array}{l}77.86 \pm 10.1 \\
131.53 \pm 65.87\end{array}$ & $\begin{array}{l}0.001 \\
0.001\end{array}$ \\
\hline $\begin{array}{l}\text { HDL-C } \\
\text { Reduced HDL-C level }\end{array}$ & $92(30.7 \%)$ & $18(16.1)$ & $74(39.4)$ & 0.000 \\
\hline Fasting blood glucose & $93.1 \pm 31.8$ & $98.36 \pm 38.43$ & $90.1 \pm 0.73$ & 0.247 \\
\hline Diabetes & $66(22)$ & $32(27.6)$ & $44(23.4)$ & 0.408 \\
\hline Hypertension & $133(44.3)$ & $67(59.8)$ & $66(35.1)$ & 0.000 \\
\hline Dyslipidemia & $176(58.7)$ & $72(64.3)$ & $104(55.3)$ & 0.127 \\
\hline MS & $115(38.3)$ & $43(38.4)$ & 78 (41.5) & 0.53 \\
\hline $\begin{array}{l}\text { GHQ } \\
<23 \\
>23\end{array}$ & $\begin{array}{l}234(78) \\
66(22)\end{array}$ & $\begin{array}{l}96(85.7) \\
16(14.3)\end{array}$ & $\begin{array}{l}138(73.4) \\
50(26.6)\end{array}$ & 0.013 \\
\hline $\begin{array}{l}\text { PSLES } \\
\text { No stress } \\
\text { Moderate stress } \\
\text { Severe stress }\end{array}$ & $\begin{array}{l}117(39) \\
141(47) \\
42(14)\end{array}$ & $\begin{array}{l}45(40.2) \\
51(45.5) \\
16(14.3)\end{array}$ & $\begin{array}{l}72(38.3) \\
90(47.9) \\
26(13.8)\end{array}$ & 0.925 \\
\hline $\begin{array}{l}\text { PHQ } \\
\text { Normal } \\
\text { Mild } \\
\text { Moderate } \\
\text { Severe }\end{array}$ & $\begin{array}{l}201(67) \\
75(25) \\
17(5.6) \\
7(2.3) \\
\end{array}$ & $\begin{array}{l}84(75) \\
23(20.5) \\
5(4.5) \\
0(-)\end{array}$ & $\begin{array}{l}117(62.2) \\
52(27.6) \\
12(6.4) \\
7(3.7)\end{array}$ & 0.05 \\
\hline
\end{tabular}

Table 3: Distribution of study participants according to mental health parameters and metabolic syndrome.

\begin{tabular}{|c|c|c|c|c|}
\hline \multirow{2}{*}{ Variable } & \multicolumn{2}{|c|}{ Metabolic Syndrome } & \multirow{2}{*}{ Total $=\mathbf{3 0 0}$} & \multirow{2}{*}{$\chi^{2}$, P value } \\
\hline & Absent $(n=185)$ & Present $(n=115)$ & & \\
\hline $\begin{array}{l}\text { Depression } \\
\text { Normal } \\
\text { Mild depression } \\
\text { Moderate depression } \\
\text { Severe depression }\end{array}$ & $\begin{array}{l}127(63.2) \\
45(60.0) \\
10(58.8) \\
3(42.9)\end{array}$ & $\begin{array}{l}74(36.8) \\
30(40.0) \\
7(41.2) \\
4(57.1)\end{array}$ & $\begin{array}{l}201(67.0) \\
75(25.0) \\
17(5.6) \\
7(2.3)\end{array}$ & $\begin{array}{l}\chi^{2}=1.39 \\
\text { P } 0.708\end{array}$ \\
\hline $\begin{array}{l}\text { General health status } \\
\text { Normal } \\
\text { Poor health status }\end{array}$ & $\begin{array}{l}146(62.4) \\
39(59.1)\end{array}$ & $\begin{array}{l}88(37.6) \\
27(40.9)\end{array}$ & $\begin{array}{l}234(78.0) \\
66(22.0)\end{array}$ & $\begin{array}{l}\chi^{2}=0.237 \\
\text { P } 0.63\end{array}$ \\
\hline $\begin{array}{l}\text { Stress } \\
\text { No stress } \\
\text { Moderate stress } \\
\text { Severe stress }\end{array}$ & $\begin{array}{l}66(56.4) \\
95(67.1) \\
24(57.1)\end{array}$ & $\begin{array}{l}51(43.6) \\
46(32.9) \\
18(42.9)\end{array}$ & $\begin{array}{l}117(39.0) \\
141(47.0) \\
42(14.0)\end{array}$ & $\begin{array}{l}\chi^{2}=4.44 \\
P 0.108\end{array}$ \\
\hline
\end{tabular}




\section{Depression}

In the present study majority of them $201(67.0 \%)$ were normal, $92(30.6 \%)$ had mild to moderate depression and only $07(2.3 \%)$ had severe depression. The prevalence of any form of depression was about 33\% among them $9.3 \%$ were men and $23.6 \%$ were women who had some form of depression. All the subjects with severe depression $100 \%$ (7) and $75.6 \%$ (28) with mild to moderate depression were females, and the difference in prevalence of depression among males and females was statistically significant $(\mathrm{p}=0.017)$. The middle aged people in the age group 31-50 years were more depressed $33.7 \%$ and $30.4 \%$. Mild to moderate depression was seen more among married people $(\mathrm{p}=0.069)$ and those living in nuclear families $(\mathrm{p}=0.074)$.

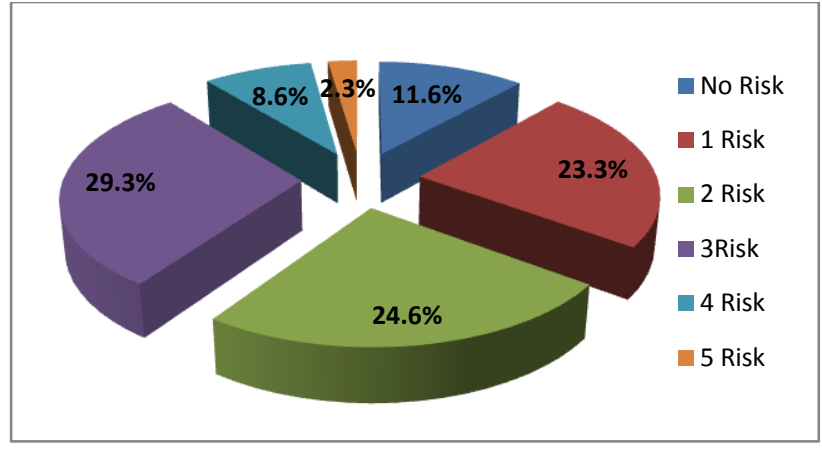

Figure 2: Distribution of study participants according to number of risk factors present.

Table 4a: Association of socio demographic factors with depression.

\begin{tabular}{|c|c|c|c|c|c|}
\hline \multirow[b]{2}{*}{ Variable } & \multicolumn{5}{|c|}{ Depression } \\
\hline & $\begin{array}{l}\text { No depression } \\
(201)\end{array}$ & $\begin{array}{l}\text { Mild-mod } \\
\text { depression (92) }\end{array}$ & $\begin{array}{l}\text { Severe depression } \\
(07)\end{array}$ & Total, $n=300$ & $\mathbf{p}$ \\
\hline \multicolumn{6}{|l|}{ Age } \\
\hline 21-30 years & $30(14.9)$ & $10(10.9)$ & $2(28.6)$ & $42(14.0)$ & \multirow{4}{*}{0.781} \\
\hline 31-40years & $59((29.4)$ & $31(33.7)$ & $2(28.6)$ & $92(30.7)$ & \\
\hline $41-50$ years & $66(32.8)$ & $28(30.4)$ & $1(14.3)$ & $95(31.7)$ & \\
\hline$>50$ years & $46(22.8)$ & $23(25)$ & $2(28.6)$ & $71(23.7)$ & \\
\hline \multicolumn{6}{|l|}{ Sex } \\
\hline Male & $84(41.8)$ & $28(30.4)$ & 0 & $112(37.3)$ & \multirow[t]{2}{*}{0.017} \\
\hline Female & $117(58.2)$ & $64(69.6)$ & $7(100)$ & $188(62.7)$ & \\
\hline \multicolumn{6}{|l|}{ Marital Status } \\
\hline Married & $166(82.6)$ & $82(89.1)$ & $5(71.4)$ & $253(84.3)$ & \multirow{3}{*}{0.069} \\
\hline Single & $30(14.9)$ & $6(6.5)$ & $1(14.3)$ & $37(12.3)$ & \\
\hline others & $5(2.5)$ & $4(4.3)$ & $1(14.3)$ & $10(3.3)$ & \\
\hline \multicolumn{6}{|l|}{ Type of school } \\
\hline Govt & $38(18.9)$ & $16(17.4)$ & $4(57.1)$ & $58(19.3)$ & \multirow{3}{*}{0.096} \\
\hline Private Aided & $69(34.3)$ & $26(28.3)$ & $2(28.6)$ & $97(32.3)$ & \\
\hline Private Unaided & $94(46.7)$ & $50(54.3)$ & $1(14.3)$ & $145(48.3)$ & \\
\hline \multicolumn{6}{|l|}{ Years of teaching } \\
\hline$<10$ years & $72(35.8)$ & $26(28.3)$ & $2(28.6)$ & $100(33.3)$ & \multirow{3}{*}{0.67} \\
\hline $10-20$ years & $77(38.3)$ & $43(46.7)$ & $3(42.9)$ & $123(41.0)$ & \\
\hline$>20$ years & $52(25.9)$ & $23(25)$ & $2(28.6)$ & $77(25.6)$ & \\
\hline \multicolumn{6}{|l|}{ Type of family } \\
\hline Nuclear & $131(65.2)$ & $60(65.2)$ & $4(57.1)$ & $195(65.0)$ & \multirow{3}{*}{0.074} \\
\hline Joint & $55(27.4)$ & $17(18.5)$ & $3(42.9)$ & $75(25.0)$ & \\
\hline Others & $15(7.5)$ & $15(16.3)$ & 0 & $30(10.0)$ & \\
\hline
\end{tabular}

\section{Metabolic syndrome and depression}

Among those who had metabolic syndrome $74(64.3 \%)$ had no depression whereas, $37(32.2 \%)$ and $4(3.5 \%)$ of the subjects had mild to moderate and severe depression respectively. This difference was statistically not significant $(p>0.05)$. Though factors like sex $(p<0.05)$, marital status and type of school and family affected the mental health status but was found statistically significant at $10 \%$ significance in the whole population and in those having MS only the type of family had significant influence $(\mathrm{p}=0.026)$.

The prevalence of metabolic syndrome $(36.8 \%, 40.2 \%$ and $57.2 \%)$ and its risk factors like BMI $(51.7 \%, 52.2 \%$ and $71.4 \%$ ), dyslipidemia $(46.8 \%, 44.4 \%$ and $57.2 \%)$, level of physical inactivity $(66.6 \%, 68.5 \% \& 85.7 \%)$, obesity $(63.2 \%, 63.1 \% \& 71.4 \%)$ and low HDL-C 
$(30.4 \%, 30.4 \%$ \& 42.8) among subjects with no depression, mild to moderate depression and severe depression respectively. Though, there is influence of depression on the metabolic syndrome risk factors, the present study could not establish statistical significance $(\mathrm{p}>0.05)$.

Table 4b: Association of metabolic syndrome risk factors with depression.

\begin{tabular}{|c|c|c|c|c|c|}
\hline \multirow[b]{2}{*}{ Variable } & \multicolumn{5}{|l|}{ Depression } \\
\hline & $\begin{array}{l}\text { No depression } \\
\text { (201) }\end{array}$ & $\begin{array}{l}\text { Mild-mod } \\
\text { depression } \\
(92)\end{array}$ & $\begin{array}{l}\text { Severe } \\
\text { depression } \\
(07)\end{array}$ & Total $n=300$ & $\mathbf{p}$ \\
\hline \multicolumn{6}{|l|}{ Physical Inactivity } \\
\hline Intense activity & $16(7.9)$ & $04(4.3)$ & 0 & $20(6.7)$ & \multirow{3}{*}{0.772} \\
\hline Moderate activity & $51(25.4)$ & $25(27.2)$ & $1(14.3)$ & $77(25.6)$ & \\
\hline Sedentary & $134(66.6)$ & $63(68.5)$ & $6(85.7)$ & $203(67.7)$ & \\
\hline \multicolumn{6}{|l|}{ Obesity (WC) } \\
\hline Normal & $74(36.8)$ & $34(36.9)$ & $2(28.6)$ & $110(36.7)$ & \multirow{2}{*}{1.0} \\
\hline Obese & $127(63.2)$ & $58(63.1)$ & $5(71.4)$ & $190(63.3)$ & \\
\hline \multicolumn{6}{|l|}{ BMI } \\
\hline Normal & $54(26.8)$ & $31(33.6)$ & 0 & $85(28.3)$ & \multirow{3}{*}{0.191} \\
\hline overweight & $43(21.4)$ & $13(14.1)$ & $2(28.5)$ & $58(19.3)$ & \\
\hline Obese & $104(51.7)$ & $48(52.2)$ & $5(71.4)$ & $157(52.3)$ & \\
\hline \multicolumn{6}{|l|}{ Systolic blood pressure } \\
\hline Normal & $130(64.7)$ & $59(64.1)$ & $6(85)$ & $195(65.0)$ & \multirow{2}{*}{0.612} \\
\hline Hypertension & $71(35.3)$ & $33(35.8)$ & $1(15)$ & $105(35.0)$ & \\
\hline \multicolumn{6}{|l|}{$\begin{array}{l}\text { Diastolic blood } \\
\text { pressure }\end{array}$} \\
\hline Normal & $145(72.1)$ & $64(69.6)$ & $6(85)$ & $215(71.7)$ & \multirow[t]{2}{*}{0.637} \\
\hline Hypertension & $56(27.8)$ & $28(30.4)$ & $1(15)$ & $85(28.3)$ & \\
\hline \multicolumn{6}{|l|}{ Triglycerides } \\
\hline Normal & $115(57.2)$ & $59(64.1)$ & $3(42.8)$ & $177(59.0)$ & \multirow{2}{*}{0.361} \\
\hline Increased & $86(42.8)$ & $33(35.9)$ & $4(57.2)$ & $123(41.0)$ & \\
\hline \multicolumn{6}{|l|}{ HDL-C } \\
\hline Normal & $140(69.6)$ & $64(69.4)$ & $4(57.2)$ & $208(69.3)$ & \multirow{2}{*}{0.813} \\
\hline Decreased & $61(30.4)$ & $28(30.4)$ & $3(42.8)$ & $92(30.7)$ & \\
\hline \multicolumn{6}{|l|}{ Diabetes } \\
\hline Absent & $159(79.1)$ & $69(75)$ & $6(85)$ & $234(78.0)$ & \multirow{2}{*}{0.644} \\
\hline Present & $42(20.8)$ & $23(25)$ & $1(15)$ & $66(22.0)$ & \\
\hline \multicolumn{6}{|l|}{ Hypertension } \\
\hline Absent & $107(53.2)$ & $46(50)$ & $4(57.2)$ & $157(52.3)$ & \multirow{2}{*}{0.838} \\
\hline Present & $94(46.7)$ & $46(50)$ & $3(42.8)$ & $143(47.7)$ & \\
\hline \multicolumn{6}{|l|}{ Dyslipidemia } \\
\hline Absent & $107(53.2)$ & $51(55.5)$ & $3(42.8)$ & $161(53.7)$ & \multirow[t]{2}{*}{0.812} \\
\hline Present & $94(46.8)$ & $41(44.4)$ & $4(57.20)$ & $139(46.3)$ & \\
\hline \multicolumn{6}{|l|}{ Metabolic syndrome } \\
\hline Absent & $127(63.2)$ & $55(59.8)$ & $3(42.8)$ & $185(61.6)$ & \multirow{2}{*}{0.518} \\
\hline Present & $74(36.8)$ & $37(40.2)$ & $4(57.2)$ & $115(38.4)$ & \\
\hline
\end{tabular}

In those having metabolic syndrome, the level of physical inactivity $(58.2 \%, 62.2 \%$ \& $75 \%)$, systolic blood pressure $(60.8 \%, 64.9 \%$ \& $25 \%)$ low HDL-C $(43.2 \%, 54.3 \%$ \& $50 \%)$, increased TGs $(72.9 \%, 64.8 \% \& 100 \%)$ and diabetes $(39.1 \%, 54.1 \%$ \& $25 \%)$ increased with the degree of depression, though the association was statistically not significant $(\mathrm{p}>0.05)$. However, increasing degree of depression was associated with BMI $(\mathrm{p}=0.053)$. 
Table 5a: Association of socio demographic factors with depression among teachers having metabolic syndrome.

\begin{tabular}{|c|c|c|c|c|c|}
\hline \multirow{2}{*}{ Variable } & \multicolumn{3}{|c|}{ Depression } & \multirow[b]{2}{*}{ Total, $n=115$} & \multirow[b]{2}{*}{$\mathbf{p}$} \\
\hline & No depression (74) & Mild-mod depression (37) & Severe depression (04) & & \\
\hline \multicolumn{6}{|l|}{ Age } \\
\hline $21-30$ years & $5(6.8)$ & - & $1(25)$ & $6(5.2)$ & \multirow{4}{*}{0.32} \\
\hline $31-40$ years & $17(23)$ & $9(24.3)$ & - & $26(22.6)$ & \\
\hline 41-51 years & $26(35.1)$ & $16943.2)$ & $1(25)$ & $43(37.4))$ & \\
\hline$>50$ years & $20(27.1)$ & $12(32.4)$ & $2(50)$ & $40(34.9)$ & \\
\hline \multicolumn{6}{|l|}{ Sex } \\
\hline Male & $31(41.9)$ & $9(24.3)$ & 0 & $40(34.9)$ & \multirow{2}{*}{0.063} \\
\hline Female & $43(58.1)$ & $28(75.3)$ & $4(100)$ & $75(65.2)$ & \\
\hline \multicolumn{6}{|l|}{ Marital Status } \\
\hline Married & $67(90.5)$ & 34 (91.9) & $4(100)$ & 105 (91.3) & \\
\hline others & $7(9.5)$ & $3(8.1)$ & - & $10(8.7)$ & \\
\hline \multicolumn{6}{|l|}{ Type of School } \\
\hline Government & $17(23)$ & $10(27.1)$ & $3(75)$ & $30(26.1)$ & \multirow{3}{*}{0.102} \\
\hline Private aided & $26(35.1)$ & 8 (21.6) & $1(25)$ & $35(30.4)$ & \\
\hline Private unaided & 31 (41.9) & $19(51.4)$ & - & $50(43.5)$ & \\
\hline \multicolumn{6}{|l|}{ Type of Family } \\
\hline Nuclear & $48(64.8)$ & $23(62.2)$ & $1(25)$ & $72(62.6)$ & \multirow{3}{*}{0.026} \\
\hline Joint & $24(32.4)$ & $8(21.6)$ & $3(75)$ & $35(30.4)$ & \\
\hline Others & $2(2.7)$ & $6(16.2)$ & - & $8(6.9)$ & \\
\hline
\end{tabular}

Table 5b: Association of metabolic syndrome risk factors with depression among teachers having metabolic syndrome.

\begin{tabular}{|c|c|c|c|c|c|}
\hline \multirow{2}{*}{ Variable } & \multicolumn{5}{|c|}{ Depression, $\mathrm{n}=115$} \\
\hline & No depression (74) & Mild-Mod depression(37) & Severe depression (04) & Total & $\mathbf{p}$ \\
\hline \multicolumn{6}{|l|}{ Physical Inactivity } \\
\hline Intense activity & $2(2.7)$ & $1(2.9)$ & - & $3(2.6)$ & \multirow{3}{*}{0.973} \\
\hline Moderate activity & $24(32.4)$ & $13(35.1)$ & $1(25)$ & $38(33.1)$ & \\
\hline Sedentary & $48(58.2)$ & $23(62.2)$ & $3(75)$ & $74(64.3)$ & \\
\hline \multicolumn{6}{|l|}{ BMI } \\
\hline Normal & $3(4.1)$ & $5(13.5)$ & - & $8(6.9)$ & \multirow{3}{*}{0.053} \\
\hline Overweight & $10(13.5)$ & $2(5.4)$ & $2(50)$ & $14(12.2)$ & \\
\hline Obese & $61(82.4)$ & $30(81.1)$ & $2(50)$ & $93(80.9)$ & \\
\hline \multicolumn{6}{|l|}{ Systolic blood pressure } \\
\hline Normal & $29(39.2)$ & $13(35.1)$ & $3(75)$ & $45(39.1)$ & \multirow{2}{*}{0.381} \\
\hline Hypertension & $45(60.8)$ & $24(64.9)$ & $1(25)$ & $70(60.9)$ & \\
\hline \multicolumn{6}{|l|}{ Diastolic blood pressure } \\
\hline Normal & $38(51.4)$ & $16(43.3)$ & $3(75)$ & $57(49.5)$ & \multirow{2}{*}{0.447} \\
\hline Hypertension & $36(48.9)$ & $21(56.7)$ & $1(25)$ & $58(50.5)$ & \\
\hline \multicolumn{6}{|l|}{ Triglycerides } \\
\hline Normal & $20(27.1)$ & $13(35.2)$ & - & $33(28.7)$ & \multirow[t]{2}{*}{0.34} \\
\hline Increased & $54(72.9)$ & $24(64.8)$ & $4(100)$ & $82(71.3)$ & \\
\hline \multicolumn{6}{|l|}{ HDL-C } \\
\hline Normal & $42(56.7)$ & $21(56.7)$ & $2(50)$ & $65(56.2)$ & \multirow{2}{*}{1.00} \\
\hline Decreased & $32(43.2)$ & $16(34.3)$ & $2(50)$ & $50(43.5)$ & \\
\hline \multicolumn{6}{|l|}{ Diabetes } \\
\hline Absent & $45(56.7)$ & $17(45.9)$ & $3(75)$ & $65(56.2)$ & \multirow{2}{*}{0.296} \\
\hline Present & $29(39.1)$ & $20(54.1)$ & $1(25)$ & $50(43.5)$ & \\
\hline \multicolumn{6}{|l|}{ Hypertension } \\
\hline Absent & $20(27)$ & $11(29.7)$ & $3(75)$ & $34(29.6)$ & \multirow[t]{2}{*}{0.135} \\
\hline Present & $54(73)$ & $26(70.3)$ & $1(25)$ & $81(70.4)$ & \\
\hline \multicolumn{6}{|l|}{ Dyslipidemia } \\
\hline Absent & $38(51.3)$ & $19(51.3)$ & $2(50)$ & $59(51.3)$ & \multirow{2}{*}{1.00} \\
\hline Present & $36(48.6)$ & 18 (48.6) & $2(50)$ & $56(48.7)$ & \\
\hline
\end{tabular}


Table 6a: Association of socio demographic factors with stress.

\begin{tabular}{|c|c|c|c|c|c|}
\hline Variable & No-mild stress (n=117) & Moderate stress (n=140) & Severe stress $(n=42)$ & Total $\mathbf{N}=\mathbf{3 0 0}$ & p \\
\hline \multicolumn{6}{|l|}{ Age } \\
\hline 21-30 years & $14(11.9)$ & $24(17.1)$ & $4(9.5)$ & $42(14.0)$ & \multirow{4}{*}{0.458} \\
\hline $31-40$ years & $32(27.3)$ & $45(32.1)$ & $14(33.3)$ & $92(30.7)$ & \\
\hline $41-50$ years & $45(38.5)$ & $37(26.4)$ & $13(30.9)$ & $95(31.7)$ & \\
\hline$>50$ years & $26(22.2)$ & $34(24.3)$ & $11(26.2)$ & $71(23.7)$ & \\
\hline \multicolumn{6}{|l|}{ Sex } \\
\hline Male & $45(38.5)$ & $50(35.7)$ & $16(38.1)$ & $112(37.3)$ & \multirow[b]{2}{*}{0.893} \\
\hline Female & $72(61.5)$ & $90(64.3)$ & $26(61.9)$ & $188(62.7)$ & \\
\hline \multicolumn{6}{|l|}{ Marital status } \\
\hline Married & $98(83.8)$ & $116(82.8)$ & $38(90.5)$ & $253(84.3)$ & \multirow{3}{*}{0.324} \\
\hline Single & $17(14.5)$ & $18(12.9)$ & $2(4.8)$ & $37(12.3)$ & \\
\hline Others & $2(1.7)$ & $6(4.3)$ & $2(4.8)$ & $10(3.3)$ & \\
\hline \multicolumn{6}{|l|}{ Type of school } \\
\hline Government & $23(19.6)$ & $26(18.6)$ & $9(21.4)$ & $58(19.3)$ & \multirow{3}{*}{0.097} \\
\hline Private aided & $40(34.2)$ & $51(36.4)$ & $6(14.3)$ & $97(32.3)$ & \\
\hline Private unaided & $54(46.1)$ & $63(45)$ & $27(64.3)$ & $145(48.3)$ & \\
\hline \multicolumn{6}{|c|}{ Years of teaching } \\
\hline$<10$ years & $33(28.2)$ & $53(37.8)$ & $13(30.9)$ & $100(33.3)$ & \multirow{3}{*}{0.554} \\
\hline $10-20$ years & $51(43.6)$ & $53(37.8)$ & $19(45.2)$ & $123(41.0)$ & \\
\hline$>20$ years & $33(28.2)$ & $34(24.3)$ & $10(23.8)$ & $77(25.6)$ & \\
\hline \multicolumn{6}{|l|}{ Type of family } \\
\hline Nuclear & $74(63.2)$ & $92(65.7)$ & $28(66.6)$ & $195(65.0)$ & \multirow{3}{*}{0.262} \\
\hline Joint & $32(27.3)$ & $30(21.4)$ & $13(30.9)$ & $75(25.0)$ & \\
\hline Others & $11(9.4)$ & $18(12.9)$ & $1(2.4)$ & $30(10.0)$ & \\
\hline
\end{tabular}

Table 6b: Association of Metabolic syndrome risk factors with Stress

\begin{tabular}{|c|c|c|c|c|c|}
\hline \multirow[b]{2}{*}{ Variable } & \multicolumn{5}{|c|}{ Stress } \\
\hline & $\begin{array}{l}\text { No-mild Stress } \\
(\mathrm{n}=117)\end{array}$ & $\begin{array}{l}\text { Moderate } \\
\text { stress } \\
(n=140)\end{array}$ & $\begin{array}{l}\text { Severe stress } \\
(n=42)\end{array}$ & Total $\mathbf{N}=\mathbf{3 0 0}$ & $\mathbf{p}$ \\
\hline \multicolumn{6}{|l|}{ Physical Inactivity } \\
\hline Intense activity & $7(6)$ & $10(7.1)$ & $3(7.1)$ & $20(6.7)$ & \multirow{3}{*}{0.881} \\
\hline Moderate activity & $31(26.5)$ & $33(23.6)$ & $26(61.9)$ & $77(25.6)$ & \\
\hline Sedentary & $79(67.5)$ & $98(70)$ & $13(30.1)$ & $203(67.7)$ & \\
\hline \multicolumn{6}{|l|}{ Obesity (WC) } \\
\hline Normal & $37(31.6)$ & $57(40.7)$ & $15(35.7)$ & $110(36.7)$ & \multirow{2}{*}{.319} \\
\hline Obese & $80(68.4)$ & $83(59.3)$ & $27(64.3)$ & $190(63.3)$ & \\
\hline \multicolumn{6}{|l|}{ BMI } \\
\hline Normal & $27(23.1)$ & $46(32.8)$ & $11(26.1)$ & $85(28.3)$ & \multirow{3}{*}{.527} \\
\hline Overweght & $24(20.5)$ & $25(17.9)$ & $9(21.4)$ & $58(19.3)$ & \\
\hline Obese & $66(56.4)$ & $69(58.9)$ & $22(52.4)$ & $157(52.3)$ & \\
\hline \multicolumn{6}{|c|}{ Systolic Blood Pressure } \\
\hline Normal & $75(64.1)$ & 97 (69.3) & $22(52.4)$ & $195(65.0)$ & \multirow[b]{2}{*}{0.129} \\
\hline Hypertension & $42(35.8)$ & $43(30.7)$ & $20(47.6)$ & $105(35.0)$ & \\
\hline \multicolumn{6}{|c|}{ Diastolic Blood pressure } \\
\hline Normal & $78(66.6)$ & $109(77.8)$ & $27(64.3)$ & $215(71.7)$ & \multirow{2}{*}{0.074} \\
\hline Hypertension & $39(33.3)$ & $31(22.1)$ & $15(35.7)$ & $85(28.3)$ & \\
\hline \multicolumn{6}{|l|}{ Triglycerides } \\
\hline Normal & $65(55.5)$ & $92(65.7)$ & $20(47.6)$ & $177(59.0)$ & \multirow{2}{*}{0.066} \\
\hline Increased & $52(44.4)$ & $48(34.3)$ & $22(52.4)$ & $123(41.0)$ & \\
\hline \multicolumn{6}{|l|}{ HDL-C } \\
\hline Normal & $80(68.4)$ & $96(68.6)$ & $32(76.2)$ & $208(69.3)$ & 0.602 \\
\hline
\end{tabular}




\begin{tabular}{|c|c|c|c|c|c|}
\hline Decreased & 37 (31.6) & $44(31.4)$ & $10(23.8)$ & $92(30.7)$ & \\
\hline $\begin{array}{l}\text { Diabetes } \\
\text { Absent } \\
\text { Present }\end{array}$ & $\begin{array}{l}93(79.5) \\
24(20.5)\end{array}$ & $\begin{array}{l}110(78.6) \\
30(21.4)\end{array}$ & $\begin{array}{l}31(73.8) \\
11(26.2)\end{array}$ & $\begin{array}{l}234(78.0) \\
66(22.0)\end{array}$ & 0.741 \\
\hline $\begin{array}{l}\text { Hypertension } \\
\text { Absent } \\
\text { Present }\end{array}$ & $\begin{array}{l}57(48.7) \\
60(51.3)\end{array}$ & $\begin{array}{l}81(57.9) \\
59(42.1)\end{array}$ & $\begin{array}{l}19(45.2) \\
23(54.8)\end{array}$ & $\begin{array}{l}157(52.3) \\
143(47.7)\end{array}$ & 0.205 \\
\hline $\begin{array}{l}\text { Dyslipidemia } \\
\text { Absent } \\
\text { Present }\end{array}$ & $\begin{array}{l}62(52.9) \\
55(47.1)\end{array}$ & $\begin{array}{l}88(62.8) \\
55(39.2)\end{array}$ & $\begin{array}{l}14(33.3) \\
28(66.6)\end{array}$ & $\begin{array}{l}161(53.7) \\
139(46.3)\end{array}$ & 0.007 \\
\hline $\begin{array}{l}\text { Metabolic syndrome } \\
\text { Absent } \\
\text { Present }\end{array}$ & $\begin{array}{l}66(56.4) \\
51(43.6)\end{array}$ & $\begin{array}{l}94(67.1) \\
46(32.8)\end{array}$ & $\begin{array}{l}24(57.1) \\
18(42.8)\end{array}$ & $\begin{array}{l}185(61.6) \\
115(38.4)\end{array}$ & 0.174 \\
\hline
\end{tabular}

Table 7a: Association of socio demographic factors with stress among teachers having MS.

\begin{tabular}{|c|c|c|c|c|c|}
\hline Variable & No mild stress $(\mathrm{n}=51)$ & Moderate stress $(n=40)$ & Severe stress $(\mathrm{n}=18)$ & Total $n=115$ & $\mathbf{p}$ \\
\hline $\begin{array}{l}\text { Age } \\
21-30 \text { years } \\
31-40 \text { years } \\
41-50 \text { years } \\
>50 \text { years }\end{array}$ & $\begin{array}{l}3(5.8) \\
10(19.6) \\
24(47.1) \\
14(27.4)\end{array}$ & $\begin{array}{l}3(7.5) \\
10(25) \\
14(35) \\
19(47.5)\end{array}$ & $\begin{array}{l}- \\
6(33.3) \\
5(27.9) \\
7(38.8)\end{array}$ & $\begin{array}{l}6(5.2) \\
26(22.6) \\
43(37.4) \\
40(34.9)\end{array}$ & 0.455 \\
\hline $\begin{array}{l}\text { Sex } \\
\text { Male } \\
\text { Female }\end{array}$ & $\begin{array}{l}20(39.2) \\
31(60.8)\end{array}$ & $\begin{array}{l}15(37.5) \\
31(77.5) \\
\end{array}$ & $\begin{array}{l}5(29.7) \\
13(72.2)\end{array}$ & $\begin{array}{l}40(34.9) \\
75(65.2)\end{array}$ & .628 \\
\hline $\begin{array}{l}\text { Marital status } \\
\text { Married } \\
\text { Single/others }\end{array}$ & $\begin{array}{l}45(88.2) \\
6(11.8)\end{array}$ & $\begin{array}{l}43 \\
3\end{array}$ & $\begin{array}{l}17(94.4) \\
1(5.6)\end{array}$ & $\begin{array}{l}105(91.3) \\
10(8.7)\end{array}$ & 0.663 \\
\hline $\begin{array}{l}\text { Type of school } \\
\text { Government } \\
\text { Private aided } \\
\text { Private unaided }\end{array}$ & $\begin{array}{l}14(27.5) \\
14(27.5) \\
23(45.1)\end{array}$ & $\begin{array}{l}12(26.1) \\
17(36.9) \\
17(36.9)\end{array}$ & $\begin{array}{l}4(22.2) \\
4(22.2) \\
10(55.5)\end{array}$ & $\begin{array}{l}30(26.1) \\
35(30.4) \\
50(43.5)\end{array}$ & 0.668 \\
\hline $\begin{array}{l}\text { Type of family } \\
\text { Nuclear } \\
\text { Joint } \\
\text { Others }\end{array}$ & $\begin{array}{l}32(62.7) \\
16(31.4) \\
3(5.8)\end{array}$ & $\begin{array}{l}31(67.4) \\
10(21.7) \\
5(10.9)\end{array}$ & $\begin{array}{l}9(50) \\
9(50) \\
-\end{array}$ & $\begin{array}{l}72(62.6) \\
35(30.4) \\
8(6.9)\end{array}$ & 0.200 \\
\hline
\end{tabular}

Table 7b: Association of Metabolic syndrome risk factors with stress among teachers having metabolic syndrome.

\begin{tabular}{|c|c|c|c|c|c|}
\hline \multirow[b]{2}{*}{ Variable } & \multicolumn{5}{|c|}{ Stress } \\
\hline & $\begin{array}{l}\text { No-mild stress } \\
(n=51)\end{array}$ & $\begin{array}{l}\text { Moderate } \\
\text { stress } \\
(n=40)\end{array}$ & $\begin{array}{l}\text { Severe stress } \\
(n=18)\end{array}$ & Total $n=115$ & $\mathbf{p}$ \\
\hline $\begin{array}{l}\text { Physical Inactivity } \\
\text { Intense activity }\end{array}$ & $2(3.9)$ & - & $1(5.5)$ & $3(2.6)$ & \\
\hline Moderate activity & $14(27.4)$ & $19(41.3)$ & $5(27.7)$ & $38(33.1)$ & 0.329 \\
\hline Sedentary & $35(68.6)$ & $27(58.7)$ & $12(66.6)$ & $74(64.3)$ & \\
\hline BMI & & & & & \\
\hline Normal & $3(5.8)$ & $5(10.5)$ & - & $8(6.9)$ & \\
\hline Overweight & $7(13.7)$ & $6(13.1)$ & $01(5.5)$ & $14(12.2)$ & 0.569 \\
\hline Obese & $41(80.4)$ & $35(76.1)$ & $17(94.4)$ & $93(80.9)$ & \\
\hline Systolic blood pressure & & & & & \\
\hline Normal & $21(41.2)$ & $19(41.3)$ & $5(27.7)$ & $45(39.1)$ & \\
\hline Hypertension & $30(52.9)$ & $21(58.7)$ & $13(72.2)$ & $70(60.9)$ & 0.561 \\
\hline $\begin{array}{l}\text { Diastolic blood pressure } \\
\text { Normal }\end{array}$ & $24(47.1)$ & $24(52.2)$ & $9(50)$ & $57(49.5)$ & 0.880 \\
\hline
\end{tabular}




\begin{tabular}{|c|c|c|c|c|c|}
\hline Hypertension & $27(52.9)$ & $22(47.8)$ & $9(50)$ & $58(50.5)$ & \\
\hline $\begin{array}{l}\text { Triglycerides } \\
\text { Normal } \\
\text { Increased }\end{array}$ & $\begin{array}{l}15(29.4) \\
36(70.6)\end{array}$ & $\begin{array}{l}16(34.8) \\
30(65.2)\end{array}$ & $\begin{array}{l}2(11.1) \\
16(88.8)\end{array}$ & $\begin{array}{l}33(28.7) \\
82(71.3)\end{array}$ & 0.168 \\
\hline $\begin{array}{l}\text { HDL-C } \\
\text { Normal } \\
\text { Decreased }\end{array}$ & $\begin{array}{l}28(54.9) \\
23(45.1)\end{array}$ & $\begin{array}{l}26(56.5) \\
20(43.5)\end{array}$ & $\begin{array}{l}11(61.1) \\
7(38.8)\end{array}$ & $\begin{array}{l}65(56.2) \\
50(43.5)\end{array}$ & 0.901 \\
\hline $\begin{array}{l}\text { Diabetes } \\
\text { Absent } \\
\text { Present }\end{array}$ & $\begin{array}{l}31(60.7) \\
20(39.2)\end{array}$ & $\begin{array}{l}23(50) \\
23(50)\end{array}$ & $\begin{array}{l}11((61.1) \\
7(38.8)\end{array}$ & $\begin{array}{l}65(56.2) \\
50(43.5)\end{array}$ & 0.515 \\
\hline $\begin{array}{l}\text { Hypertension } \\
\text { Absent } \\
\text { Present }\end{array}$ & $\begin{array}{l}13(25.5) \\
38(74.5)\end{array}$ & $\begin{array}{l}17(36.9) \\
29(63.1)\end{array}$ & $\begin{array}{l}4(22.2) \\
14(77.7)\end{array}$ & $\begin{array}{l}34(29.6) \\
81(70.4)\end{array}$ & 0.354 \\
\hline $\begin{array}{l}\text { Dyslipidemia } \\
\text { Absent } \\
\text { Present }\end{array}$ & $\begin{array}{l}26(51.1) \\
25(49.1)\end{array}$ & $\begin{array}{l}29(63.1) \\
17(36.9)\end{array}$ & $\begin{array}{l}4(22.2) \\
14(77.7)\end{array}$ & $\begin{array}{l}59(51.3) \\
56(48.7)\end{array}$ & 0.013 \\
\hline
\end{tabular}

\section{DISCUSSION}

The present study also is similar to the earlier studies wherein the influence of depression and stress on metabolic syndrome is evident, but a statistical association could not be established. Though, the study showed that risk of MS increased with age and the risk factors were significantly associated with MS. TG, low HDL-C and BMI were found to be independent predictors of Metabolic Syndrome among the teachers.

According to the study the prevalence of any form of depression was about $33 \%$ in the present study. $9.3 \%$ of men and $23.6 \%$ of women had some form of depression; however those having severe depression were all females (100\%).

The metabolic syndrome and some of its components like obesity and dyslipidemia was higher in subjects with increasing severity of depression, however was statistically not significant. Similarly Nicole V et al. study did not find any statistically significant association between the severity of depressive symptoms and metabolic syndrome (OR 1.20, 95\%-CI=1.02-1.41). However, large waist circumference, high triglycerides levels and low HDL levels were significantly associated with higher cortisol levels in the depressed group $(\beta=0.178, p=0.02 ; \beta=0.247, p=0.001 ; \beta=-0.156, p=0.04$, respectively). ${ }^{7}$

Viola Vaccarino et al revealed the number of metabolic syndrome risk factors increased gradually across the three depression categories ( $p$ 0.003) in women with suspected coronary artery disease. Hypertension, triglyceride levels and waist circumference were those most strongly associated with depression and those with depression had two times risk of metabolic syndrome. ${ }^{21}$

Similarly the study among police officers in New York city showed that depression added to the risk of obesity
$(\mathrm{OR}=1.34)$, triglyceride $(\mathrm{OR}=1.476)$, glucose intolerance $(\mathrm{OR}=1.567)$ and hypertension $(\mathrm{OR}=1.518) .{ }^{6}$ However, our study showed that depression added to the risk of having Metabolic syndrome $(\mathrm{OR}=1.21)$, diabetes mellitus $(\mathrm{OR}=1.21)$, hypertension $(\mathrm{OR}=1.14)$ and physical inactivity $(\mathrm{OR}=2.05)$.

A meta-analysis by Bariana Mazuk shows that depression is associated with a $60 \%$ increase in risk of developing type 2 diabetes rivals other known risk factors for this disease. ${ }^{22}$ According to the Mac Andrew systematic review of metabolic syndrome and insulin resistance increases 6-7 fold rise in diabetes incidence. ${ }^{5}$ The present study shows that the odds for developing diabetes with depression is about $21 \%$ (OR 1.211 , 95\% CI $=0.972-$ 1.59). Thus, with the presence of depression will add to the risk of diabetes.

In the present study, $43.6 \%$ of the subjects without stress, $32.9 \%$ and $42.9 \%$ with mild and severe stress were observed to have Metabolic Syndrome ( $p>0.05)$. 13.8\% and $14.4 \%$ of females and males had severe stress and $47.9 \%$ and $45.0 \%$ of the females and males among the study subjects had moderate stress. The odds for developing metabolic syndrome with any form of stress (OR 1.425, 95\% CI $=0.982-1.868)$. The Whitehall II study, showed people with chronic work stress were twice likely to develop metabolic syndrome than those without work stress $(\mathrm{OR}=2.25,95 \% \mathrm{CI}=1.31-3.85) .{ }^{8}$

The present study also shows a higher odds for obesity (OR 1.415, 95\% CI= 1.166-1.664), triglycerides (OR $1.28,95 \% \mathrm{CI}=1.04-1.52$ ), hypertension (OR $1.28,95 \%$ $\mathrm{CI}=1.04-1.52$ ), though the association was not statistically significant. Stressful life events related to finance, health and workload were associated with insulin resistance, obesity and triglycerides had a higher odds for having IGT however, stress did not associate significantly with IFG, HDL cholesterol, or blood pressure. ${ }^{9,10}$ 
However the present study showed that higher level of stress was associated with higher prevalence of systolic hypertension $(52.9 \%, 58.7 \% \& 72.2 \%)$, increased TGs $(70.6 \%, 65.2 \%$ \& $88.8 \%)$ dyslipidemia $(49.1 \%, 36.9 \%$ \& $77.7 \%)$ and diabetes $(39.2 \%, 50.0 \%$ \& 38.8\%) among those with metabolic syndrome. Stress was not significantly associated with any of the risk factors identified in the present study population.

However, the study showed similar trend in prevalence of depression and stress, risk factors of metabolic syndrome among those with some form of depression and stress, though the association between could not be statistically established except for stress and dyslipidemia.

\section{CONCLUSION}

The earlier research has established that there is increase in occurrence of life stress before the onset of major depression and also metabolic syndrome as an intermediate stage between depression and CVD. The current study has a prevalence of moderate to severe depression, stress and the risk factors components of MS was similar to other populations. The poor mental health status was significantly high among females and was associated with high fasting blood glucose levels. Though, the present study did not show any statistical association between the mental health status and the occurrence of MS or its components except severity of stress was related to dyslipidemia and depression with obesity. It clearly indicates that the risk factors are influenced by stress and depression, which are a necessary evil of modernization and urbanization of the communities.

As nearly $50-60 \%$ of the mortality in the developing countries is due to NCDs especially CVDs, affecting the younger and economically productive population. This not only increases the cost of health care in turn increases the burden on weak health care systems and the economy as a whole. Thus, workplace screening of the employees will help in creating a platform for creating awareness introduction of lifestyle modification interventions. The screening for NCDs should include evaluation of mental health parameters as well, for holistic approach to health. These efforts into primary prevention by the developing countries will not only be cost effective, they will help in developing sustainable communities.

The studies that have correlated the stress and depression with metabolic syndrome are based on blood levels of cortisol, thus there is a need to further explore the hypothesis based on questionnaire such that it can be part of screening programmes and feasible for low and middle income countries.

Funding: No funding sources Conflict of interest: None declared

Ethical approval: The study was approved by the Institutional Ethics Committee

\section{REFERENCES}

1. Chatterjee R, Arora M. Life events and psychiatric disorders. Mental health reviews. Available at Http://Www.Psyplexus.Com/Mhr/.Html>. Accessed on $08 / 09 / 2012$.

2. Alberti G, Zimmet P. Workshop of the IDF Task Force on epidemiology and prevention. International Diabetes Federation consensus worldwide definition of the metabolic syndrome. 2006;1-12.

3. Sawant A, Mankeshwar R, Shah S, Raghavan R, Dhongde G, Raje H et al. Prevalence of metabolic syndrome in urban India. Cholesterol. 2011;1-7.

4. Brunner EJ, Hemingway H, Walker BR, Page M, Clarke P, Juneja MJ et al. Adrenocortical autonomic and inflammatory causes of the metabolic syndrome: nested case control study. Circulation. 2002;106:2659-65.

5. Cornier M, Dabelea D, Hernandez TL, Lindstrom RC. The metabolic syndrome. Endocrine Reviews. 2008;29(7):777-822.

6. Hartley TA, Knox SS, Fekedulegn D, BarbosaLeiker C, Violanti JM, Michael E. Association between depressive symptoms and metabolic syndrome in police officers: results from two crosssectional studies. J Environment Pub Health. 2012;2012:1-9.

7. Vogelzangs N, Kristen S, Ferrucci L, Simonsick EM, Ble A, Schrager M, et al. Hypercholestermic depression- is associated with the metabolic syndrome in late-life. Psychoneuroendocrinology 2007;32(2):151-9.

8. Deepa M, Farooq S, Datta M, Deepa R, Mohan V. Prevalence of metabolic syndrome using WHO, ATPIII and IDF definition in Asian Indians: the Chennai Urban Rural Epidemiology Study (CURES 34). Diabetes metabolism research and review. 2007;23(2):127-34.

9. Chandola T, Burnner E, Marmot M. Chronic stress at work and the metabolic syndrome: prospective study. BMJ. 2006;32(7540):521-5

10. Antti JP, Katri R, Tiinamaija T, Eriksson JG, Leif G, Isomaa Bo. Stressful life events and metabolic syndrome. the prevalence, prediction and prevention of diabetes (PPP)- botnia study. Diabetes Care. 2010;33:378-84.

11. Nagai M, Tsuchiya KJ, Toulopoulou T, Takei N. Poor mental health associated with job dissatisfaction among school teachers in Japan. J Occup Health. 2007;49:515-22.

12. Claro S, Bedragal P. Mental health status of teachers in 12 schools of Puente Alto, Santiagio, Chile. Rev Med Chil. 2003;131(2):159-67.

13. Samad NIA, Hashim Z, Moin S, Abdullah H. Assessment of stress and its risk factors among primary school teachers in the Klang Valley, Malaysia. Glob J Health Sci. 2010;2(2):163-71.

14. Desai A, Tandon N. Challenges in prevention and management of diabetes mellitus and metabolic syndrome in India. Curr Sci. 2009;97:356-66. 
15. World Health Organization. WHO STEPS surveillance manual: The WHO STEP wise approach to chronic disease risk factor surveillance. Geneva: World Health Organization; 2005.

16. Martin A, Rief W, Klaiberg K, Braehler B. Validity of the brief patient health questionnaire mood scale (PHQ-9) in the general population. Gen Hosp Psychiatry. 2006;28(1):71-7.

17. Instruction manual instructions for Patient Health Questionnaire (PHQ-9) and GAD-7 measures. Pifffzer. 2007.

18. Sengupta P, Benjamin AI. Psychosocial factors in migrant population study. Health and PopulationPerspectives and Issues. 2004;27(1):17-28.

19. Goldberg DP, Gater R, Sartorius N, Ustun TB, Piccinelli $\mathrm{M}$, Gureje $\mathrm{O}$ et al. The validity of two versions of the GHQ in the WHO study of mental illness in general health care. Psychol Med. 1997;27:191-7.

20. Goldberg DP, Williams P. Manual of the general health questionnaire. Windsor, England: NFER Publishing; 1978.

21. Vaccarino V, McClure C, Johnson BD, Sheps DS, Bittner V, Rutledge $\mathrm{T}$ et al. Depression, the metabolic syndrome and cardiovascular risk. Psychosom Med. 2008;(70):40-8.

22. Mezuk B, William W, Albrecht S, Golden SH. Depression and type 2 diabetes over the lifespan. Diabetes Care. 2008;31(12):2383-90.

Cite this article as: Narayanappa S, Manjunath R, Kulkarni P. Mental health risk factors influencing metabolic syndrome among secondary school teachers of Mysore city, India. Int J Community Med Public Health 2016;3:998-1009. 